\title{
Prevalência da periodontite crônica em pacientes atendidos em uma clínica escola de odontologia
}

\author{
Prevalence of chronic periodontitis in \\ patients attended in a dental school clinic
}

\author{
Karolayne da Silva Sousa' ${ }^{1}$ (i) \\ Lara Niely Bezerra Leal ${ }^{2}$ (1) \\ João Lucas de Sena Cavalcante ${ }^{3}$ (1) \\ Luciana Mara Peixoto Araújo4 (1)
}

\author{
${ }^{1,2,4}$ Centro Universitário Leão Sampaio (Juazeiro do Norte). Ceará, Brasil. karolayne.sousa03@gmail.com, \\ laranielybezerra@outlook.com, lucianamara@leaosampaio.edu.br \\ ${ }^{3}$ Autor para correspondência. Centro Universitário Leão Sampaio. Juazeiro do Norte, Ceará, Brasil. joao.lucasdesena@hotmail.com
}

\begin{abstract}
RESUMO | INTRODUÇÃO: A periodontite crônica é uma das patologias bucais mais comuns, apresentando elevadas taxas de perda dentária. OBJETIVO: identificar a prevalência da periodontite crônica dos pacientes atendidos em um centro universitário no interior do Ceará. METODOLOGIA: Trata-se de uma pesquisa do tipo transversal, cujas informações da população pesquisada foram obtidas por meio de prontuários dos pacientes atendidos no período de 2013 a 2018. Foram incluídos na pesquisa dois mil, oitocentos e vinte e um prontuários que continham as informações completas necessárias ao estudo e o TCLE (Termo de Consentimento Livre Esclarecido) devidamente assinado. As variáveis analisadas foram idade, sexo, tipo de doença periodontal, doença sistêmica e hábito de fumar. RESULTADOS: Entre os pacientes avaliados foi observado que $21,5 \%$ destes apresentaram periodontite crônica. O sexo feminino apresentou maior prevalência com 55,8\% e a faixa etária mais acometida por essa doença foi entre 3044 anos. Dos pacientes fumantes $29 \%$ desenvolveram a patologia periodontal, e aqueles com alterações sistêmicas, 25,2\% apresentaram periodontite crônica, sendo a doença cardiovascular a de maior prevalência com 57,2\%. CONCLUSÃo: Entre os pacientes avaliados $21,5 \%$ apresentaram periodontite crônica, sendo de grande valia entender como se dá a distribuição e os demais fatores que estão associados a essa doença, pois esses dados podem ser utilizados no planejamento de ações voltadas a saúde pública.
\end{abstract}

PALAVRAS-CHAVE: Doença periodontal. Periodontite Crônica. Epidemiologia. Prevalência.
ABSTRACT | INTRODUCTION: Chronic periodontitis is one of the most common oral pathologies, with high rates of tooth loss. OBJECTIVE: to identify the prevalence of chronic periodontitis in patients attended at a university center in the interior of Ceará. METHODOLOGY: This is a cross-sectional research, whose information of the researched population was obtained from the medical records of patients attended in the period from 2013 to 2018. It was included two thousand, eight hundred and twenty-one medical records that contained the complete information required for the study and the Informed Consent Form (ICF) duly signed. The variables analyzed were: age, sex, type of periodontal disease, systemic disease and smoking habit. RESULTS: Among the evaluated patients, it was observed that $21.5 \%$ of them had shown chronic periodontitis. The female had a higher prevalence with $55.8 \%$ and the age group most affected by this disease was between 30-44 years. Among the smoking patients, 29\% developed periodontal pathology, and those with systemic changes, $25.2 \%$ had chronic periodontitis, with cardiovascular disease being the most prevalent with $57.2 \%$. CONCLUSION: Among the evaluated patients, $21.5 \%$ had chronic periodontitis, and it is of great value to understand how the distribution and other factors are associated with this disease, thus this data can be used in planning actions aimed to public health.

KEYWORDS: Periodontal disease. Chronic periodontitis. Epidemiology. Prevalence. 


\section{Introdução}

A periodontite crônica é conceituada como uma doença inflamatória que provoca injúria as estruturas que sustentam o dente (cemento, osso alveolar e ligamento periodontal) caracterizada pelo acúmulo do biofilme onde a microbiota está mais comumente presente nas superfícies subgengivais. A velocidade da destruição dos tecidos depende da qualidade dessa microbiota e da resposta do hospedeiro, sendo esta mais prevalente em indivíduos adultos, devido a sua progressão lenta|.

A periodontite crônica é uma das patologias bucais mais comuns, apresentando elevadas taxas de perda dentária. Uma grande parcela da população sofre de algum tipo de doença periodontal, podendo iniciar em uma idade precoce, e sem uma intervenção adequada, pode levar a perda do elemento dentall.

Essa patologia manifesta-se clinicamente como uma inflamação gengival, caracterizada pela presença de sangramento à sondagem, redução da resistência dos tecidos periodontais e perda de inserção dos tecidos de suporte. Esse tipo de periodontite apresenta aspectos variáveis como: edema ou recessão gengival, lesão de furca e mobilidade dental. A causa primordial é a presença de microrganismos presentes no biofilme, aderidos na superfície dos dentes associado a uma deficiência na resposta inflamatória do indivíduol.

De acordo com Llanosf a forma crônica da doença periodontal apresenta um desenvolvimento lento, de forma que essa condição dificilmente é encontrada em pacientes jovens. Esta apresenta-se de maneira localizada ou generalizada, quando acomete menos de $30 \%$ dos sítios afetados presentes na cavidade bucal ou em mais de $30 \%$ dos sítios afetados, respectivamente.

Conforme visto no estudo de Benoist te crônica apresenta fatores que quando associados tornam o individuo mais susceptível ao seu desenvolvimento, sendo estes classificados como fatores ambientais (presença de bactérias patogênicas específicas), comportamentais (hábito de fumar) e biológicos (doenças sistêmicas) que de acordo com a sua gravidade podem gerar um impacto negativo na qualidade de vida desses indivíduos, limitando as atividades funcionais bucais e psicossociais.

No âmbito da saúde oral, Demmer e Papapanou局, relatam que o número de estudos sobre a prevalência de periodontite ainda é escasso. Segundo Benoist periodontite crônica é mais prevalente nos homens e a sua progressão e gravidade está intimamente ligada com a presença de fatores de risco. Demmer e Papapanou|fobservaram que a prevalência da doença aumenta com o avanço da idade, assim como a severidade da mesma. Pode-se compreender que a prevalência da periodontite varia consideravelmente de acordo com o perfil demográfico da população, sendo necessários estudos de maior abrangência.

A forma crônica da periodontite se inicia a partir de uma gengivite e sua evolução se dá de forma lenta, na qual a perda de inserção e osso alveolar serão verificados subsequentemente. O avanço da doença é contínuo e a resposta pode ser influenciada pelos seguintes fatores: idade; aumento da perda de inserção e da bolsa periodontal; fatores retentivos de placa; virulência da microbiota colonizada, tabaco e algumas alterações sistêmicasł.

Através do estudo de Machadol verifica-se inúmeros fatores de risco para a periodontite crônica, e por esta ser uma doença bastante presente na população, torna-se importante identificar esses grupos de risco e assim diminuir a sua prevalência.

O presente estudo teve como objetivo identificar a prevalência da periodontite crônica correlacionando com a faixa etária e alterações sistêmicas nos pacientes atendidos na clínica odontológica de um centro universitário do interior do Ceará. 


\section{Metodologia}

\section{Caracterizações da pesquisa}

Diante dos objetivos propostos foi realizado um estudo epidemiológico retrospectivo de dados secundários do tipo quantitativo que analisou a prevalência da periodontite crônica nos pacientes atendidos no Centro universitário Doutor Leão Sampaio (Unileão) no interior do Ceará.

Este tipo de estudo permite ter um maior conhecimento a respeito da condição de saúde periodontal da região sul do Ceará.

\section{População da pesquisa}

A população para qual realizamos este estudo foi obtida por meio de prontuários de pacientes que foram atendidos no período do primeiro semestre de 2013 ao primeiro semestre de 2019 na clínica escola de odontologia da Unileão.

\section{Amostra da pesquisa}

A presente pesquisa contou com a análise de 8040 prontuários que após a avaliação dos critérios de inclusão e exclusão, foram incluídos 2821 prontuários.

\section{Critérios de inclusão}

Foram incluídos na pesquisa apenas os prontuários que continham as informações completas sobre anamnese, exame intra e extra oral, índices orais: índice de placa; índice de sangramento gengival estabelecido por Löe e Silness (1963); exame periodontal simplificado e periograma (nos casos em que se faz necessário). E, além disso, possuíam o TCLE devidamente assinado pelo paciente.

\section{Critérios de exclusão}

Foram excluídos da pesquisa os prontuários que continham dados incompletos ou que apresentaram difícil compreensão.

\section{Variáveis da pesquisa}

As variáveis analisadas foram idade, sexo, diagnóstico da doença periodontal alterações sistêmicas e hábito de fumar. O diagnóstico periodontal foi obtido através dos exames: índice de placa; índice de sangramento gengival estabelecido por Löe e Silness (1963); exame periodontal simplificado e periograma, ambos registrados nos prontuários avaliados. Os critérios para diagnóstico de gengivite e periodontite foram dados a partir dos resultados de cada exame estudado.

Para o diagnóstico da periodontite crônica observouse presença de perda de inserção e bolsas periodontais verdadeiras registradas no periograma. Para diagnóstico de gengivite, foi analisado o índice de sangramento gengival, observando as devidas porcentagens (considerando $>10 \%$ alteração da normalidade, indicativo de gengivite). Associado ao índice de sangramento, foi avaliado o índice de placa, considerando $>30 \%$ como indesejado.

\section{Instrumentos da pesquisa}

Prontuários de pacientes que foram atendidos na clínica escola de odontologia e a tabela da coleta de dados (Quadro 1) que continham as variáveis necessárias para a realização da pesquisa. 


\begin{tabular}{|c|c|c|c|c|c|}
\hline $\begin{array}{c}N^{\circ} \text { DO } \\
\text { PRONTUÁRIO }\end{array}$ & CONDIÇÃO PERIODONTAL: & SEXO & IDADE & FUMANTE & $\begin{array}{l}\text { ALTERAÇÃO } \\
\text { SISTÊMICA }\end{array}$ \\
\hline & SAÚDE( ) G( )PC( )PA() & $F() M()$ & & $\begin{array}{l}\text { SIM( ) NÃO( ) } \\
\text { FOI ( ) }\end{array}$ & $\begin{array}{l}\text { SIM ( ) Qual? } \\
\text { NÃO ( ) }\end{array}$ \\
\hline & SAÚDE ( ) G( )PC ( )PA( ) & $F() M()$ & & $\begin{array}{l}\text { SIM( ) NÃO( ) } \\
\text { FOI ( ) }\end{array}$ & $\begin{array}{l}\text { SIM ( ) Qual? } \\
\text { NÃO ( ) }\end{array}$ \\
\hline & SAÚDE ( ) G( )PC( )PA( ) & $F() M()$ & & $\begin{array}{l}\text { SIM( ) NÃO( ) } \\
\text { FOI ( ) }\end{array}$ & $\begin{array}{l}\text { SIM ( ) Qual? } \\
\text { NÃO ( ) }\end{array}$ \\
\hline & SAÚDE ( ) G( )PC ( )PA( ) & $F() M()$ & & $\begin{array}{l}\text { SIM( ) NÃO( ) } \\
\text { FOI ( ) }\end{array}$ & $\begin{array}{l}\text { SIM ( ) Qual? } \\
\text { NÃO ( ) }\end{array}$ \\
\hline & SAÚDE( ) G( )PC( )PA( ) & $F() M()$ & & $\begin{array}{l}\text { SIM( ) NÃO( ) } \\
\text { FOI ( ) }\end{array}$ & $\begin{array}{l}\text { SIM ( ) Qual? } \\
\text { NÃO ( ) }\end{array}$ \\
\hline & SAÚDE ( ) G( )PC( )PA( ) & $F() M()$ & & $\begin{array}{l}\text { SIM( ) NÃO( ) } \\
\text { FOI ( ) }\end{array}$ & $\begin{array}{l}\text { SIM ( ) Qual? } \\
\text { NÃO ( ) }\end{array}$ \\
\hline & SAÚDE ( ) G( )PC ( )PA( ) & $F() M()$ & & $\begin{array}{l}\text { SIM( ) NÃO( ) } \\
\text { FOI ( ) }\end{array}$ & $\begin{array}{l}\text { SIM ( ) Qual? } \\
\text { NÃO ( ) }\end{array}$ \\
\hline & SAÚDE ( ) G( )PC ( )PA( ) & $F() M()$ & & $\begin{array}{l}\text { SIM( ) NÃO( ) } \\
\text { FOI ( ) }\end{array}$ & $\begin{array}{l}\text { SIM ( ) Qual? } \\
\text { NÃO ( ) }\end{array}$ \\
\hline & SAÚDE ( ) G( )PC ( )PA( ) & $F() M()$ & & $\begin{array}{l}\text { SIM( ) NÃO( ) } \\
\text { FOI ( ) }\end{array}$ & $\begin{array}{l}\text { SIM ( ) Qual? } \\
\text { NÃO ( ) }\end{array}$ \\
\hline & SAÚDE ( ) G( )PC ( )PA( ) & $F() M()$ & & $\begin{array}{l}\text { SIM( ) NÃO( ) } \\
\text { FOI ( ) }\end{array}$ & $\begin{array}{l}\text { SIM ( ) Qual? } \\
\text { NÃO ( ) }\end{array}$ \\
\hline & SAÚDE ( ) G( )PC ( )PA( ) & $F() M()$ & & $\begin{array}{l}\text { SIM( ) NÃO( ) } \\
\text { FOI ( ) }\end{array}$ & $\begin{array}{l}\text { SIM ( ) Qual? } \\
\text { NÃO ( ) }\end{array}$ \\
\hline & SAÚDE ( ) G( )PC ( )PA( ) & $F() M()$ & & SIM( ) NÃO( ) FOI ( ) & $\begin{array}{l}\text { SIM ( ) Qual? } \\
\text { NÃO ( ) }\end{array}$ \\
\hline & SAÚDE ( ) G( )PC ( ) PA( ) & $F() M()$ & & SIM( ) NÃO( ) FOI ( ) & $\begin{array}{l}\text { SIM ( ) Qual? } \\
\text { NÃO ( ) }\end{array}$ \\
\hline
\end{tabular}

\section{Procedimentos da pesquisa}

O procedimento da pesquisa foi realizado em uma sala, onde a análise dos prontuários foi feita por quatro examinadores calibrados de forma independente, verificando cuidadosamente as informações contidas nos exames periodontais. A coleta dos dados foi realizada no período de outubro de 2018 a abril de 2019.

A calibração dos quatro examinadores foi realizada com a orientação de uma especialista em periodontia, que juntos analisaram todos os critérios de inclusão e exclusão e em seguida avaliaram 20 prontuários a fim de tirar quaisquer dúvidas, observando cada exame (Índice periodontal simplificado, periograma, índice de sangramento gengival e índice de placa visível) e expondo todos os critérios e normativas padrões de cada exame, a fim de haver uma unanimidade na avaliação dos prontuários.

\section{Análise estatística}

Os dados coletados pelos pesquisadores foram tratados de forma a se fazer um levantamento estatístico descritivo, quantificando aspectos que fossem passivos deste tipo de categorização. Foi feito o uso de gráficos para correlacionar o comportamento das variáveis estudadas nesta pesquisa.

\section{Aspectos éticos}

A pesquisa foi submetida e aprovada pelo Comitê de Ética e Pesquisa sob o número CAAE 89734218.6.0000.5048. Os riscos da pesquisa foram mínimos, nos quais os dados que foram coletados envolveram apenas o que realmente foi de interesse para a pesquisa, assegurando a preservação da identidade do paciente, não permitindo que o mesmo seja exposto. Os dados de identificação pessoal não foram coletados na pesquisa, mantendo assim, em conformidade com a resolução 466/2012 do Conselho Nacional de Saúde. 


\section{Resultados}

Neste estudo foram avaliados 8040 prontuários no período de outubro de 2018 a abril de 2019, onde após a análise dos critérios de inclusão e exclusão adotados, foram incluídos nessa pesquisa 2821 prontuários, dos quais 817 (29\%) apresentaram saúde periodontal e 2004 (71\%) doença periodontal, conforme gráfico 1 abaixo.

Gráfico 1. Percentual de pacientes que apresentaram saúde e doença periodontal

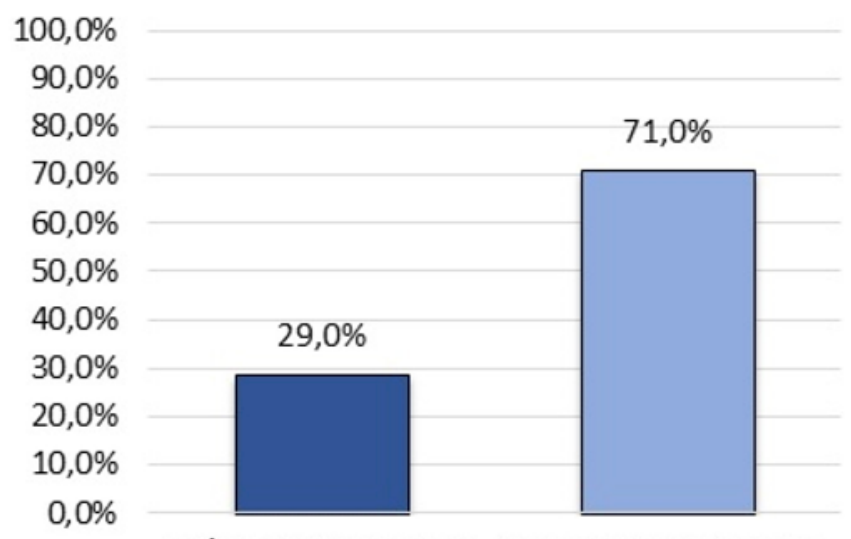

SAÚDE PERIODONTAL DOENÇA PERIODONTAL

Fonte: Dados da pesquisa (2019).

Com relação aos pacientes que possuíam doença periodontal, a gengivite mostrou-se mais prevalente com $1567(78,2 \%)$ quando comparada a periodontite crônica com $430(21,5 \%)$ e a periodontite agressiva com 7 (0,3\%), de acordo com o gráfico 2 .

Gráfico 2. Percentual de pacientes da amostra que apresentaram gengivite e periodontite crônica

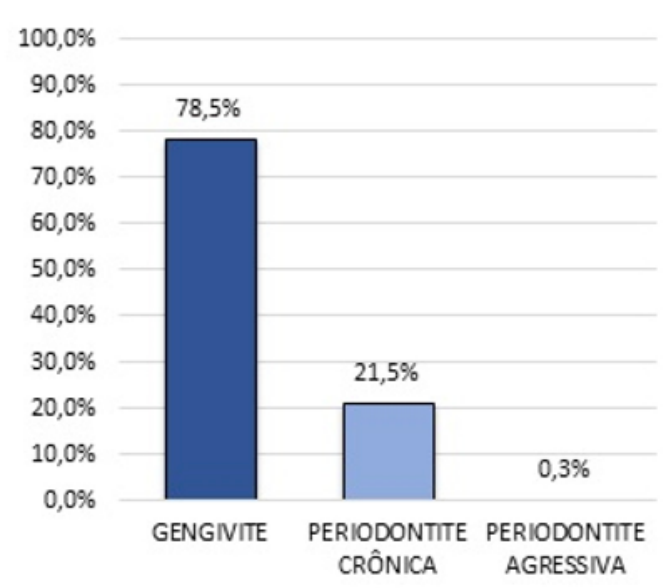

Fonte: Dados da pesquisa (2019).
Na população pesquisada (430 pacientes) apresentaram-se com periodontite crônica, nos quais foi observado maior prevalência no sexo feminino com 240 (55,8\%), enquanto que o sexo masculino demostrou menor prevalência com 190 (44,2\%), dados no gráfico 3.

Gráfico 3. Percentual de pacientes com periodontite crônica em relação ao sexo

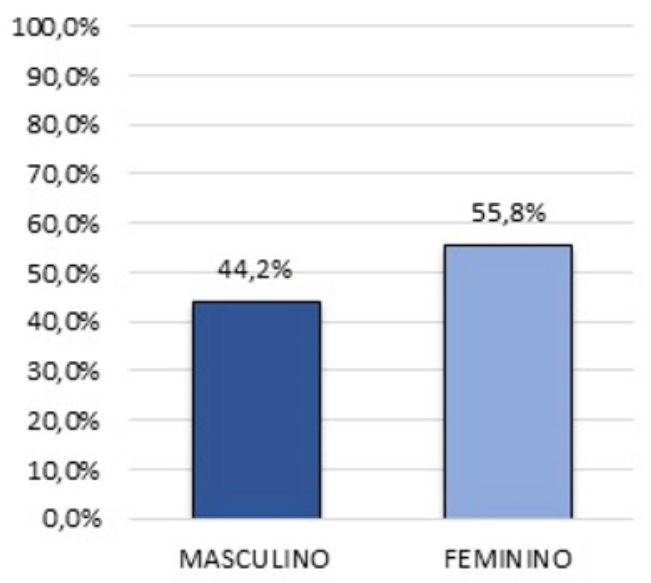

Fonte: Dados da pesquisa (2019).

No que se refere a faixa etária, a amostra estudada e identificada com periodontite crônica foi dividida em 4 grupos etários: I grupo 15-19 anos; II grupo 20-29 anos; III 30-44 anos e IV grupo 45-65 anos, onde verificou-se maior prevalência da periodontite crônica nos pacientes com idade entre 30-44 anos, representando 219 (50,9\%) dos pacientes com a doença periodontal.

Gráfico 4. Percentual da distribuição etária dos pacientes com periodontite crônica.

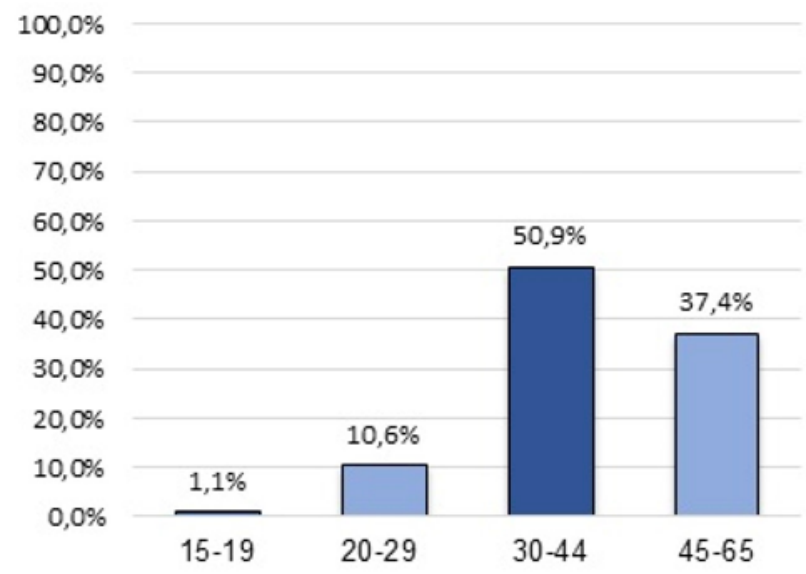

Fonte: Dados da pesquisa (2019). 
Analisando a correlação entre a periodontite crônica e o tabagismo na amostra estudada, $604(21,4 \%)$ pacientes declararam ser fumantes. Destes, 175 (29\%) apresentaram periodontite crônica.

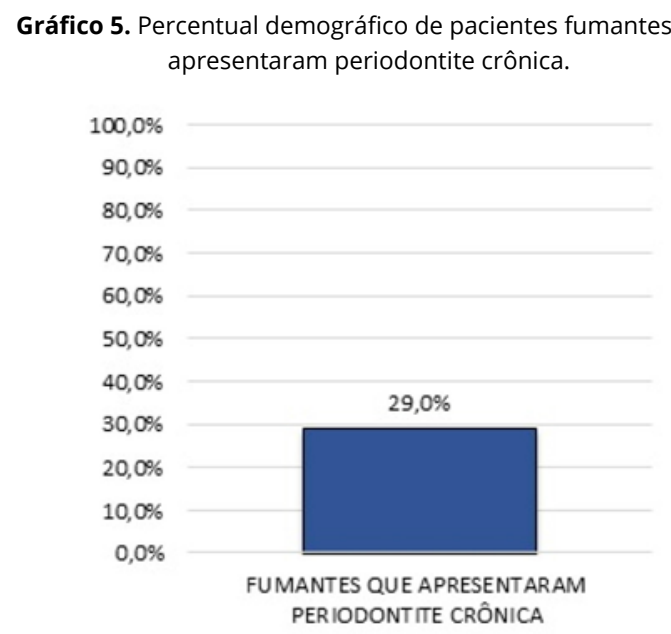

Fonte: Dados da pesquisa (2019).

Quando se verificou a associação entre as doenças sistêmicas e a periodontite crônica, 433 pacientes apresentaram algum tipo de alteração sistêmica, dos quais 110 (25,2\%) apresentaram periodontite crônica, de acordo com gráfico 6.

Gráfico 6. Percentual de pacientes com doença sistêmica que apresentaram periodontite crônica

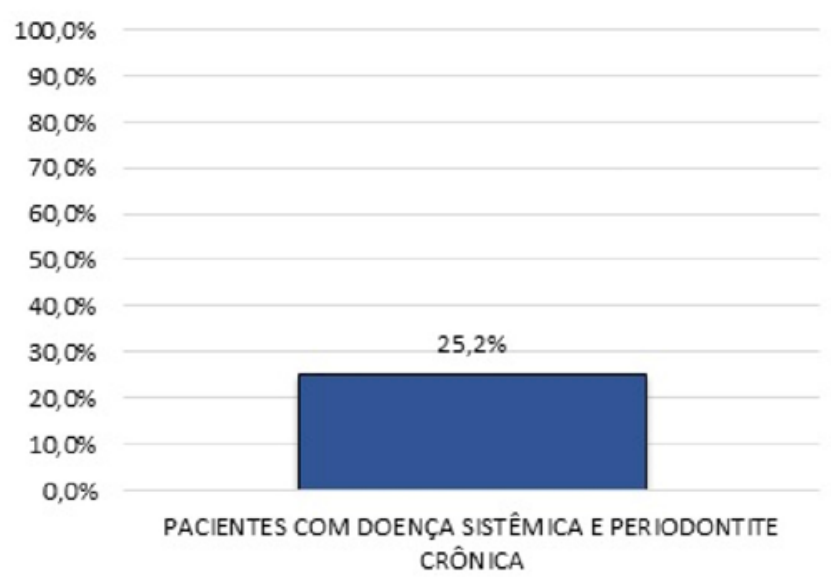

Fonte: Dados da pesquisa (2019).
De acordo com os resultados da pesquisa, 110 pacientes apresentaram alguma doença sistêmica e periodontite crônica. Indivíduos com doença cardiovascular exibiram maior prevalência da periodontite crônica quando comparados às demais condições sistêmicas, representando 63 pacientes (57,2\%), seguida respectivamente da diabetes $17(15,4 \%)$, depressão 9 $(8,1 \%)$, distúrbio de tireoide $4(3,6 \%)$, doença renal e doença reumática $2(1,8 \%)$ dentre outras diferentes condições sistêmicas 13 (12,1\%), abaixo no gráfico 7 .

Gráfico 7. Percentual das doenças sistêmicas de maior prevalência em
pacientes com periodontite crônica

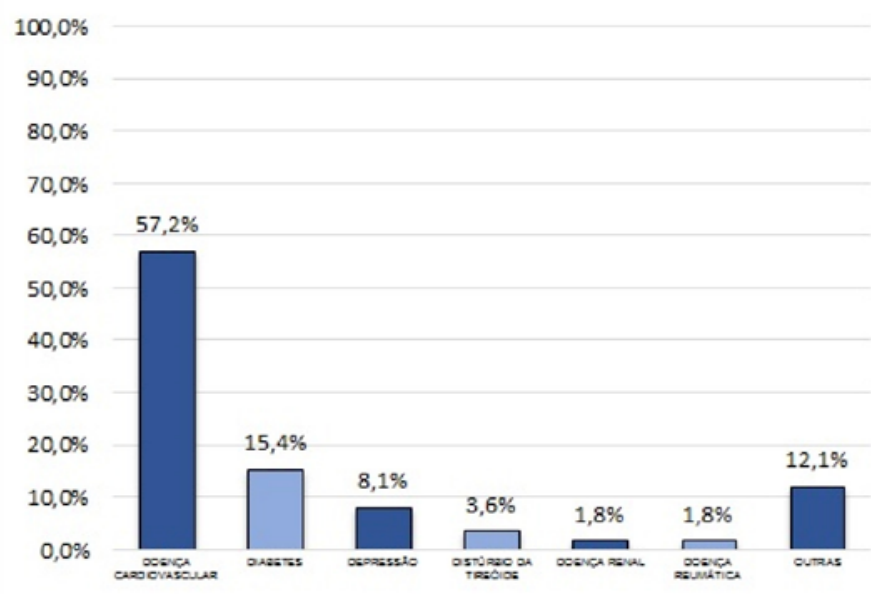

Fonte: Dados da pesquisa (2019).

\section{Discussão}

Atualmente as doenças periodontais ainda se mostram bastante comuns na população. Nesse contexto foi observado a necessidade de identificar a prevalência dessa doença em nossa região. Dessa forma encontrou-se maior prevalência das doenças periodontais nos indivíduos estudados, em comparação a saúde periodontal, corroborando com o estudo de Drumond-Santana onde foram avaliados $167 \mathrm{pa}$ cientes, dos quais $15,7 \%$ apresentaram periodonto saudável e os outros $84,3 \%$ apresentaram algum tipo de doença periodontal.

As patologias periodontais mais prevalentes neste estudo foram a gengivite e a periodontite crônica, sendo a doença gengival a de maior prevalência em relação a periodontite crônica, semelhante ao estudo de Melo円, que analisou 233 prontuários, no qual a amostra evidenciou que a gengivite possui maior prevalência com $55 \%$ diferenciando da periodontite crônica com $45 \%$. 
Apesar de o resultado estar de acordo com outras pesquisas a menor prevalência da doença periodontal crônica pode estar relacionada aos critérios de exclusão estabelecidos, como também às características regionais e culturais da população estudada.

Várias análises epidemiológicas denotam que tanto a gengivite como a periodontite crônica acometem mais frequentemente homens do que mulheres?. 0 inverso foi observado neste estudo, pois o sexo feminino expressou maior prevalência da periodontite crônica quando comparado ao sexo masculino. Este achado corrobora com o estudo de Souza e Taba ${ }^{12}$, onde foram avaliados 2273 pacientes, cujo sexo feminino apresentou um maior percentual da periodontite com $64,5 \%$ enquanto que o sexo masculino retratou $35,5 \%$.

Apesar de as mulheres parecerem procurar com maior frequência o cirurgião dentista, estas podem estar mais propensas ao desenvolvimento de doenças periodontais, devido às constantes alterações hormonais, podendo interferir na saúde geral e bucal.

A periodontite crônica é considerada uma patologia comum, que pode ser observada em quase todas as faixas etárias, porém é comumente observada em adultos e idosos, tendo sua prevalência e severidade elevadas de acordo com a idade. Nesse estudo o grupo etário de maior prevalência foi encontrado no grupo III (30-44 anos), apresentando menor prevalência no grupo I (15-19 anos), verificando que a idade pode significar um fator de risco para a periodontite crônica. Na análise de Souza e Taba用 podese observar que o grupo etário de maior prevalência desta patologia encontra-se na terceira e quarta décadas de vida com $56,89 \%$ ratificando os resultados deste estudo.

Em conformidade com o trabalho de Machadol compreende-se que a periodontite crônica apresenta uma progressão lenta na destruição dos tecidos periodontais, que sem uma intervenção adequada pode levar a perda do elemento dentário. Visto que a prevalência da mesma cresce ao longo do tempo, a idade torna-se um importante fator de risco para o desenvolvimento da patologia periodontal.
Segundo Meuzel e Piazza国 a análise de seus dados revelou que pacientes fumantes apresentam maior risco de desenvolver periodontite em comparação a pacientes não fumantes. Em contrapartida, a população a qual foi realizado este estudo apontou resultados divergentes à literatura, pois pode-se observar que somente um terço dos pacientes fumantes apresentaram periodontite crônica.

Esse resultado pode ser atribuído à frequência e intensidade do tabagismo, que interfere na resposta imunológica do hospedeiro, aumentando assim a chance de desenvolver alterações periodontais. Quando há uma menor frequência e intensidade do hábito, menores serão as chances de desenvolver periodontite crônica 4 o que pode ter ocorrido com a população do estudo.

Ao analisar fatores associados a doença periodontal com presença de doenças crônicas, entende-se que ambas as patologias compartilham determinantes locais e sistêmicos. Alguns trabalhos relatam que fatores de origem sistêmica como diabetes tipo I e tipo II tornam os individuos mais susceptíveis ao desenvolvimento de patologias periodontais'15.

Nesse cenário, podemos dizer que a saúde geral e o desenvolvimento de algumas doenças sistêmicas podem influenciar nas infecções periodontais, como exemplo de alterações sistêmicas: a diabetes, distúrbios hormonais ocorridos durante o ciclo menstrual, doenças cardiovasculares e uso de alguns medicamentos relacionados a determinadas condições'国.

Os resultados obtidos nesta pesquisa apontaram que dos pacientes com doenças sistêmicas cerca de um quarto apresentaram periodontite crônica. No entanto, os achados dessa pesquisa não podem comprovar que não há uma associação entre as doenças periodontais com as alterações sistêmicas, pois tratam-se de dados coletados através de informações prestadas pelos próprios pacientes, podendo os mesmos omitir este tipo de informação. 
Dentre os pacientes com periodontite crônica e portadores de doenças sistêmicas, a doença que obteve maior prevalência foram as doenças cardiovasculares, seguida da diabetes. Similar aos resultados encontrados na pesquisa de Szpilman用, onde os pacientes que apresentaram somente hipertensão como alteração sistêmica associada a periodontite crônica exibiram maior prevalência da doença periodontal com $60,7 \%$, enquanto que os pacientes que apresentaram hipertensão e diabetes simultaneamente manifestaram 39,3\%.

De acordo com Szpilman喝, a literatura faz uma ligação entre a diabetes e as patologias periodontais. Uma maior prevalência, extensão e também gravidade da doença periodontal foi comprovada em pacientes que possuem diabetes através de várias pesquisas, podendo ainda ter associação com o controle metabólico. Sua pesquisa também detectou que as doenças do periodonto pode ser considerada um fator de risco para patologias cardiovasculares, e ainda que os fatores sistêmicos possam estar relacionados de maneira conjunta na etiologia de ambas as doenças.

\section{Conclusão}

Tendo em vista os aspectos observados:

- Houve maior prevalência de pacientes com doenças periodontais que indivíduos com saúde periodontal, dentre estes a gengivite apresentou maior prevalência, seguida da periodontite crônica e periodontite agressiva.

- Foi verificado maior prevalência na periodontite crônica do sexo feminino, em pacientes com faixa etária entre 30-44 anos e quando correlacionado as alterações sistêmicas a doença cardiovascular foi a de maior prevalência. Na população estudada foi observado menor prevalência da periodontite crônica em pacientes fumantes e com alterações sistêmicas.

É de grande valia entender como se dá a distribuição e os demais fatores que estão associados a essa doença, pois esses dados podem ser utilizados no planejamento de ações voltadas a saúde pública. Diante da importância desses dados é necessária a realização de mais estudos em diferentes regiões, de forma padronizada para obter resultados mais precisos.

\section{Contribuições dos autores}

Sousa KS, Leal LNB realizaram a coleta de dados da pesquisa e escrita do manuscrito. Cavalcante JLS realizou a coleta de dados da pesquisa e análise estatística. Araújo LMP realizou coleta de dados da pesquisa e a escrita do manuscrito realizando a revisão do mesmo embasada na literatura.

\section{Conflitos de interesses}

Nenhum conflito financeiro, legal ou político envolvendo terceiros (governo, empresas e fundações privadas, etc.) foi declarado para nenhum aspecto do trabalho submetido (incluindo, mas não se limitando a subvenções e financiamentos, participação em conselho consultivo, desenho de estudo, preparação de manuscrito, análise estatística, etc.).

\section{Referências}

1. Minaya-Sánchez M, Medina-Solís CE, Maupomé G, VallejosSánchez AA, Casanov-Rosado JF, Marquez-Corona ML. Prevalence of and Risk Indicators for Chronic Periodontitis in Males from Campeche. Rev Salud Pública. 2007;9(3):388-98. doi: 10.1590/ s0124-00642007000300007

2. Singh A, Agarwal V, Tuli A, Khattak BP. Prevalence of chronic periodontitis in Meerut: A cross sectional survey. J Indian Soc Periodontol. 2012;16(4):529-32. doi: 10.4103/0972-124X.106895

3. Nuto SAS, Nations MK, Costa ICC. Aspectos culturais na compreensão da periodontite crônica: um estudo qualitativo. Cad Saúde Pública. 2007;23(3):681-690. doi: 10.1590/S0102311X2007000300026

4. Llanos AH, Silva CGB, Ichimura KT, Rebeis ES, Giudicissi M, Romano MM et al. Impact of aggressive periodontitis and chronic periodontitis on oral health-related quality of life. Braz Oral Res. 2018;32:e006. doi: 10.1590/1807-3107bor-2018.vol32.0006

5. Benoist HM, Seck-Diallo A, Diouf A, Yabbre S, Sembene M, Diallo PD. Profile of chronic and aggressive periodontitis among Senegalese. J Periodontal Implant Sci. 2011;41(6):279-284. doi: 10.5051/jpis.2011.41.6.279

6. Demmer R, Papapanou PN. Epidemiologic Patterns Of Chronic And Aggressive Periodontitis. Periodontol 2000. 2010;53:28-44. doi: $10.1111 / \mathrm{j} .1600-0757.2009 .00326 . \mathrm{x}$

7. Machado V, Botelho J, Amaral A, Proença L, Alves R, Rua J et al. Prevalence and extent of chronic periodontitis and its risk factors in a Portuguese subpopulation: a retrospective crosssectional study and analysis of Clinical Attachment Loss. Peer J. 2018;6:e5258. doi: $10.7717 /$ peerj.5258 
8. Zimmermann GS, Brum RS. Condição Periodontal de Pacientes com Relato de Doenças e/ou Eventos Cardiovasculares Tratados nas Clínicas Odontológicas da Universidade Federal de Santa Catarina. [dissertação]. Santa Catarina: Universidade Federal de Santa Catarina; 2015.

9. Drumond-Santana T, Costa FO, Zenóbio EG, Soares RV, Santana TD. Impacto da doença periodontal na qualidade de vida de indivíduos diabéticos dentados. Cad Saúde Pública. 2007;23(3):637-644. doi: 10.1590/S0102-311X2007000300022

10. Melo SMA, Oliveira VM, Ferreira TBC, Monturil LL, Oliveira PRD, Roriz VM. Avaliação do perfil periodontal dos pacientes atendidos na faculdade de odontologia da Unievangélica. Braz J Periodontol 2016;26(2):7-13.

11. Machion L, Freitas PM, Cesar Neto JB, Nogueira Filho GR, Nociti Jr FH. A influência do sexo e da idade na prevalência de bolsas periodontais. Pesq Odont Bras. 2000;14(1):33-37. doi: 10.1590/ S1517-74912000000100007

12. Souza SLS, Taba Jr M. Cross-sectional Evaluation of Clinical Parameters to Select High Prevalence Populations for Periodontal Disease. The Site Comparative Severity Methodology. Braz Dent J. 2004;15(1):46-53. doi: 10.1590/S0103-64402004000100009

13. Meuzel Drdzv, Piazza MM. Periodontite Crônica Em Pacientes Adultos Fumantes E Não Fumantes. J Oral Invest. 2013;2(2):9-13. doi: 10.18256/2238-510X/j.oralinvestigations.v2n2p9-13

14. Khan S, Khalid T, Awan KH. Chronic periodontitis and smoking: Prevalence and dose-response relationship. Saudi Med J.

2016;37(8): 889-94. doi: 10.15537/smj.2016.8.14223

15. Lorenzo SM, Alvarez R, Andrade E, Piccardo V, Francia A, Massa $F$ et al. Condições periodontais e fatores associados em adultos e idosos: resultados da primeira Pesquisa Nacional de Saúde Bucal no Uruguai. Cad Saúde Pública. 2015;31(11):2425-2436. doi: 10.1590/0102-311X00012115

16. Szpilman ARM, Silva LR, Sylvestre NC, Coutinho Junior EZ, Silva RS, Endringer DC. Condição periodontal de hipertensos e diabéticos: impacto da atuação da equipe de saúde da família. HU Revista. 2012, 38(1-2):67-73. 